\title{
O desafio de implementar as ações de saúde do trabalhador no SUS: a estratégia da RENAST
}

\author{
The challenge of implementing Workers' \\ Health actions in the Brazilian National \\ Health System (SUS): The RENAST
}

Elizabeth Costa Dias 1

Maria da Graça Hoefel 2

$1_{\text {CESTEH/Fiocruz. }}$

Av. Leopol do Bulhões 1480

Manguinhos, 21041-210,

Rio de JaneiroRJ.

bethdias@ensp.fiocruz.br

2 Coordenação de Saúde

do Trabalhador

COSAT/DAPE/SAS,

Mnistério da Saúde.
Abstract This paper deals with the "model" of workers' health care del ivery based on the stra tegy of a National Network for Comprehensive Workers' Health Care (RENAST), which is under construction within the National Health System (SUS) in Brazil. RENAST is a national network of information and health practices, developed with the main purpose of providing health care action, health surveillanceand health promotion actions, at the primary health care level, medium and high complexity outpatient clinics and hospital level, under social control. According to this "model", "Workers' Health Reference Centers" play a role of technical support, and dissemination of a "culture" of the co rerole pl ayed by wo rk in the health/dise ase process, and the "culture" of social production of disease. Also, these "Reference Cen ters" h ave to play a vital role in negotiations and political/institutional arrangements with other social acto rs, both within the Health Sector and with other institutional actors, within their geographic jurisdiction. This paper includes a bri ef summary of Wo rkers' Health inception and development in the National Health System (SUS) of B razil; some crucial opera tional aspects of this development process; and, finally, some advancements, difficulties and perspectives for this new model, based on the RENAST.

Key words Workers' health, Workers' health care, Occupational health
Resumo A construção da Rede de Atenção In tegral à Saúde do Trabalhador (RENAST) no SUS, pa ra implementar as ações de uma atenção diferenciada pa ra os trabalhadores na rede de serviços de saúde é a principal estra tégia adotada pela área Técnica de Saúde do Trabalhador do Ministério da Saúde (COSAT), a partir de janei ro de 2003. A RENAST está organizada como rede nacional de informação e práticas de saúde: ações assistenciais, de vigilância e promoção da saúde, nas linhas de cuidado da atenção básica, da média e alta co mpl exidade ambula to rial, pré-hospitalar e hospitalar, sob o controle social, nos três níveis de gestão do SUS. Na proposta da RENAST, os Cen tros de Referência em Saúde do Trabalhador (CRST) dei xam de ser porta de en trada e assumem o papel de suporte técnico, pólo irradiador da cultura da centralidade do tra balho e produção social das doenças, e lócus de pactuação das ações de saúde, intra e interseto rialmente, no seu território de abrangência. Neste texto, são apresentados: uma breve retrospectiva do processo de insti tuição das ações de Saúde do Trabalhador no âmbito do SUS, a descrição dos aspectos organizacionais e operacionais da RENAST, nos Estados e municípios e iden tificados avanços, dificuldades e as perspectivas vislumbradas.

Palavras-chave Saúde do trabalhador, Atenção à saúde, Políticas de saúde do trabalhador 


\section{Introdução}

A organização da atenção à saúde da população reflete o acúmulo técnico-científico e a correlação das forças sociais, que se expressam em uma dada política de saúde. Segundo Merhy, Cecílio e Nogueira (1992), a organização da produção de serviços de saúde se dá a partir do arranjo de saberes da área, bem como das estratégias políticas implementadas por um determinado grupo social. Dessa forma, o "modelo" tem uma dimensão assistencial e tecnológica e se ex pressa como proj eto político, articu1 ado a determinadas forças sociais.

Este tex to tem por objetivo descrever o "m odelo" de atenção à saúde dos trabalhadores no Si s tema Único de Saúde (SUS), adotado na con s trução da Rede de Atenção In tegral à Sa úde do Trabalhador (RENAST) nos serviços de saúde, envo lvendo técnicos, trabalhadores e o movimento social organizado. O processo de preparação da III Conferência Nacional de Saúde do Trabalhador (3a CNST) constitui uma oportunidade privil egiada de reflexão sobre a instituição do campo daSaú de do Trabalhador, a Política Nacional de Saúde do Trabalhador (PNST)e, em particular, pa radiscussão da a tenção integral aos trabalhadbres, com base na estratégia da RENAST.

$\mathrm{Na}$ abordagem do tema, são apresentados: uma breve retro s pectiva da insti tuição das ações de Sa ú de do Trabalhador no âmbi to do SUS, em s eus aspectos con ceituais e políti cos e identificados os avanços, as dificuldades enfrentadas, além de algumas pers pectivas vislumbradas.

\section{An tecedentes}

A organização de uma atenção diferenciada à saúde dos trabalhadores surge, no mundo ocidental, no século 18, na Ingla terra, com a Revolução Industrial. Pressionados pelos prejuízos econômicos, decorrentes dos altos índices de aciden tes e adoecimen to determinados pelas péssimas condições de vida e trabalho e pelas reivindicações dos trabalhadores por mudanças, industriais da época passaram a contratar médicos, a tribuindo-lhes a responsabilidade de "cuidar" da saúde dos trabalhadores. A recom endação de Ba ker, médico inglês, reflete o espírito da prática nascente da medicina do trabalho, em 1883:

Coloque no interior da sua fábrica o seu próprio médico que servirá de intermediário entre você, os seus trabalhadores e o público. Deixe-o visitar a fábrica, sala por sala, sempre que existam pessoas trabalhando, de maneira que ele possa verificar o efei to do trabalho sobre as pessoas. E se ele verificar que qualquer dos trabalhadores está sofrendo a influência de causas que possam ser prevenidas, a ele competirá fazer tal prevenção. Dessa forma você poderá dizer: meu médico é minha defesa, pois a ele dei toda a minha auto ridade no que diz respei to à proteção da saúde e das condições físicas dos meus operários; se algum deles vi er a sofrer qualquer alteração da saúde, o médico é que deve ser responsabilizado (Men des \& Dias, 1991).

Sob a égi de da medicina do trabalho eram, e ainda são, desenvolvidas práticas assistenciais, de cunho biologicista, dirigidas essencialmente à seleção e manutenção da higidez da força de trabalho, com ações cen tradas no ambiente e no posto de trabalho, sendo o traba$1 \mathrm{~h}$ ador apenas obj eto dessas ações.

Na primeira metade do século 20, mudanças nos processos produtivos decorren tes das duas grandes guerras mundiais e os esforços de recon s trução pós-guerra deram forma a novos problemas e necessidades de saúde relacionadas ao trabalho. Outros profissionais se juntaram à equipe médica, enfocando aspectos da higiene, da ergonomia e da segurança do trabalho, conformando a prática da Saúde Ocupacional. Apesar da abord a gem multidisciplinar e da ampliação do foco das ações de saúde, estas perman eceram cen tradas no ambiente de trabalho, atrel adas aos parâmetros dos "limites de tolerância" con s i derados "científicos e seg uros", permanecendo o trabalhador um objeto das ações.

Nos anos 50 do século passado, a melh oria das condições de vida das pessoas, sob a égi de de políticas públicas desenhadas pelo estado de bem-estar social e a reorganização dos movimentossociais, particularmen te na Eu ropa, permi ti ram a em ergência de novos questionamentos sobre as condições de trabalho e reivindicações de mudanças capazes de garantir saúde e melhorar o ambi en te e a qualidade de vida das pessoas.

$\mathrm{Na}$ Itália, o movim en to pela Reforma Sanitária, que envo lveu os trabalhadores organizados, técnicos de serviços de saúde e das instituições de ensino e pesquisa, tro u xe à cena política as condições de trabalho geradoras de doença e a necessidade de modificá-las. Os trabalhadores assumiram a con dução da luta por melhores condições de trabalho e de vida, sob 
o princípio da "não delegação" (Berlinguer, 1983; Oddone, 1986).

E cos desse movimento chegaram ao Brasil em meados dos anos 70, em mom en to histórico marc ado pelo processo de reor ganização social, o fim da ditadu ra militar e redem ocratização no país. Com o apoio dos trabalhadores, em particular dos sindicatos sintonizados com as premissas do "novo sindicalismo" e as formulações teórico - con ceituais produzidas pela epidemiologia social, a agenda da Reforma Sanitária Brasileira incluiu as questões de Saúde do Trabalhador.

Assim, o movim en to da Sa ú de do Trabalhador, no Brasil, toma forma no final dos anos 70, tendo como eixos: a defesa do direito ao trabalho digno e saudável; a participação dos trabalhadores nas decisões sobre a organização e gestão dos processos produtivos e a busca da ga ra ntia de atenção integral à saúde. Desde então, a implementação de ações de assistência e de vigilância da saúde para os trabalhadores, pela rede pública de serviços de saúde, vem contribuindo para a instituição da Saúde do Trabalhador no país (Dias, 1994).

Os Programas de Saúde do Trabalhador (PST) de senvo lvi dos na rede pública de serviços de saúde, por sindicatos de trabalhadores e hospitais universitários, foram direcionados para desvelar o adoecimen to relacionado ao trabalho e incorporar a participação dos trabalhadores, valorizan doseus rel a tos sobre as condições de trabalho, considerando-os sujeitos das ações de saúde e dando visibilidade ao problema na sociedade (Augusto, 1985; Mendes, 1988; Pimenta \& Capistrano, 1988; Costa et al., 1989). A incorporação dessas questões nas práticas co tidianas de sindicatos de trabalhadores mais organizados foi reforçada pelas assessorias de saúde e pela criação, em São Paulo, do Departamen to Intersindical de Estudos e Pesquisas de Saúde e dos Ambientes de Trabalho (DIESAT) (Ribei ro \& Lacaz, 1984). Essas inicia tivas se espalharam pelo país, alimentandoo processo social de discussão e organização da luta pela melhoria das condições de vida e de trabalho digno e saudável, alicerces do movimen to da Sa ú de do Trabalhador (Lacaz, 1996).

A VIII Con ferência Nacional de Saúde, em março de 1986, contribu iupara reafirmar à sociedade brasileira a saúde como direito de cidadania e dever do Estado provê-la. Em dezembro do mesmo ano, foi realizada a I Con ferência Nacional de Saúde do Trabalhador que $\mathrm{m}$ a roou politicamen te o movimen to da insti- tuição desse campo (Mnistério da Saúde/Fiocruz, 1987).

A Consti tuição Federal de 1988 incorporou as questões de Sa ú de do Trabalhador ao enu nciar o con ceito ampliado de Saúde, incluindo entre seus determinantes - as condições de alimentação, habitação, educação, renda, meio ambiente, trabalho, em prego - e ao atribuir ao SUS a responsabilidade de coordenar as ações no país. Essa atribuição foi regulamentada, em 1990, pela lei 8.080 que definiu os princípios e a formatação do SUS. Consolidava-se assim, no plano legal e institucional, o campo da Saúde do Trabalhador.

En tret an to, um árduo caminho se desenhava à fren te para fazer cumprir a lei e inserir as ações de Sa ú de do Trabalhador no SUS. Trad icionalmente, no Brasil, a atenção à saúde dos trabalhabres inseri dos no setor formal de trabalho - a regulação e inspeção das condições de trabalho e a assistência médica - era atribu ição dos Ministérios do Trabalho e da Previdência Social. Apesar das inovações, o tex to con s titucional manteve a superposição ou con corrência de algumas dessas atribuições, fomentando con $\mathrm{fl}$ i tos en tre os setores, particularmente quan to às ações de fiscalização dos ambientes e condições de trabalho.

A Saúde do Trabalhador também foi formalmente incorporada no organograma e práticas do Ministério da Sa ú de (MS) e nos níveis estaduais e municipais do SUS. Entretanto, desde o início, a compartim entalização das estruturas vem dificultando a implem entação de uma atenção integral e integrada aos trabalhadores. Ao lon go desses 15 anos, a Sa ú de do Trabalhador tem sido colocada ora no âmbito da estrutura da Vigilância, ora na Assistência ou em outras instâncias administrativas do SUS, dependen do do form a to institu ci onal vigente.

Porém, essas dificuldades não impediram que houvesse avanços na inserção de ações de Saúde do Trabalhador no SUS. Em 1991, o MS apre s en tou à soci ed ade, a primei ra proposta de um Plano de Trabalho em Saúde do Trabalhador no SUS (MS, 1991). A estratégia definida à época privilegi ava a organização das ações em Centros de Referência em Saúde do Trabalhador (CRST), como alternativa para potencializar os escassos recursos disponíveis, facilitar o diálogo com o movimen to social e capacitar profissionais para desenvo lver as ações propo stas. A II Conferência Naci onal de Sa ú de do Trabalhador, realizada em 1994, foi um momen to importante de mobilização técnica e política, e 
palco de acirrada disputa quanto aos espaços de atuação dos Ministérios do Trabalho e da Saúde (MS, 2002).

Nos anos 90, a Área Técnica de Saúde do Trabalhador do Ministério da Saúde (COSAT) con cen trou esforços em um amplo processo de ca pacitação técnica, para as ações de Vigilância e na Atenção Básica da Saúde; a elaboração de pro tocolos, normas e diretrizes, en tre as quais, a Norma Operac i onal de Saú de do Trabalhador (NOST); a Lista de Doenças Relacionadas ao Trabalho e o Manual de Procedimentos para ori entar as ações de Saúde do Trabalhador na rede de serviços de saúde, publicado em 2001; o de senvolvim en to de indicadores de saúde do trabalhador para os Sistemas de Informação em Saúde, em especial a Rede Intera gencial de Informações para a Saú de (RIPSA) e uma proposta de Política Nacional de Saúde do Trabalhador, colocada em con sulta na socied ade, em 2001.

Finalizandoesta breve retrospectiva, podese dizer que os anos 90 foram férteis para a consolidação do campo da Sa ú de do Trabalhador no país. Além das atividades de s envo lvidas por distintos setores sociais, o trabalho dos CRST, na rede de serviços de saúde, con tribu iu pa ra a construção de uma atenção diferenciada à saúde dos trabalhadbres no SUS, como expressão da luta organizada dos trabalhadores por melhores condições de vida e trabalho e da ação de tutela da saúde dos cidadãos pelo Estado.

En tret an to, a par dos avanços, também podem ser iden tific adas dificuldades, en tre elas: o pequeno número e a distribuição desigual dos CRST nos Estados e municípios; a baixa cobertu ra das ações, que não alcançavam um número expressivo de trabalhadores; a desarticulação da atuação do con jun to de ações de saúde, perm a n ecen do à margem das estrutu ras e políticas do SUS, conformando os "guetos da Saúde do Trabalhador", além de uma fraca articulação inters etori al.

Assim, 15 anos após a regulamentação da a tri buição con stitu cional da atenção integral à saúde dos trabalhadores pela Lei Orgânica da Saúde 8.080/90 e das ex periências implem en tadas na rede pública de serviços de saúde, podese dizer que o SUS ainda não inco rpo rou, de fo $r$ ma efetiva, em suas concepções, paradigmas e ações, o lugar que o "trabalho" ocupa na vida dos indivíduos e suas relações com o espaço sócioambiental. Ou seja: o papel do "trabalho" na determinação do processo saúde/ doença dos trab alh adores diret a m en te envo lvi dos nas ativida- des produtivas, da população em geral e nos impactos ambientais que essas atividades produ zem (Hoefel, Dias \& Si lva, 2005).

Apesar de não se ter, ainda, uma reflexão mais or ganizada sobre essas questões e sem minimizar as questões intestinas, próprias do SUS, é importante destacar, en tre os fatores que têm dificultado a concretização desses objetivos, a emergência do processo de reestrutu ração produtiva. Um rela tivo distanciamen to históri co já permite perceber que, nos anos 90, quando ainda "comemorávamos" as con quistas do movimen to da Saúde do Trabalhador e se buscavam formas para efetivá-las, tom avam corpo, noBrasil, mudanças radicais na forma de organizar e gerenciar o trabalho e de reorientar as ações do Estado, na perspectiva do chamado “Estado mínimo", em sintonia com um proce sso mais global.

Entre as conseqüências dessas mudanças, p a ra o trabalho, o modo de vida e as condições de saúde da população, dos trabalhadores em particular, podem ser identificados: o desemprego estrutural; a precarização do trabalho e ocrescimen to do setor informal, com perda de direitos trabalhistas e previdenciários historicamente conquistados pelos trabalhadores. O desemprego estrutural tem repercutido na organização dos sindicatos, obrigan do-os a deslocar o foco de atuação para a preservação de pos tos de trabalho, deixando em segundo plano as condições em que o mesmo é execut ado. As relações de solidariedade e cooperação no cotidiano de trabalho, essenciais para a saúde e segurança dos trabalhadores, são substituídas pela competição predatória, socialmente valorizada e estimulada pelos meios de comunicação.

O perfil epidemiológi co do adoecimen to dos trabalhadores também reflete essas mudanças, traduzindo-se no aumen to da prevalência de doenças relacion adas ao trabalho, como as Lesões por Esforços Repetitivos (LER) ou Distúrbios Osteomusculares Relacionados ao Trabalho (DORT), que respon dem por cerca de $80 \%$ dos casos atendidos nos CRST, na atualidade e em formas de adoecimento mal c a racterizadas, entre elas o estresse, a fadiga física e mental e outras expressões de sofrimento relacionadas ao trabalho. Como agravan te, obs erva-se que essas "novas" formas de adoecimen to convivem, no país, com as doenças profissionais clássicas como a silicose, as intoxicações por metais pesados e por agrotóxicos, entre outras (MS, 2004). 
Nesse cenário, a clientela atendida pelos CRST dei xou de ser, predominantemente, encaminhada pelos sindicados e pela Previdência Social passando a ser con s ti tuída maciçamente por trabalhadores provenien tes do setor informal, precá rio ou por de sem pregados. A su stentação para a organização e gerenciamento dos CRST, propiciada pela participação dos sindicatos de trabalhadores, também ficou muito prejudicada. Os técnicos dos CRST têm encontrado dificuldades para lidar com essa nova realidade. Observa-se um descompasso entre as "n ova s" necessidades e demandas dos trabalhadores e as práticas de saúde construídas anteriormente. Nas ações de Vigilância dos ambien tes e condições de trabalho pelas equipes dos CRST, por exemplo, a parece claramente a con tradição en tre as exigências técnicas de mudança dos processos de trabalho, que, em muitos casos, significam interdição e a luta dos trabalhadores pela manutenção do emprego. É gran de também a dificuldade dos profissionais para lidar com os "n ovo s" probl emas de saúde doença dos trabalhadores, entre eles, as manifestações de sofrimento psíquico, os efeitos à ex posição a baixas do sa gens de substâncias tóxicas e às novas formas de organização e gestão do trabalho. Estes são alguns exemplos das dificuldades en frentadas na atenção à Saúde do Trabalhador no SUS.

Apesar da escassez e inconsistência das informações sobre a situação de saúde dos trabalhadores, no Brasil, segundo o IBGE, a população econ omicamente ativa (PEA) é composta por aproximadam en te 85 milhões de trabalhadores, dos quais apenas 23 milhões, cerca de um terço, são cobertos pelo Seg u ro de Acidentes do Trabalho (SAT). E s tima-se que mais de um milhão de trabalhadores são atingidos, a cada ano, por acidente ou agravo rel aci on ado ao trabalho (MS, 2004). Entre 1999 a 2003, a Previdência Social regis trou 1.875.190 aciden tes de trabalho, s en do 15.293 com óbi tos e 72.020 com incapacidade permanente, com uma média de 3.059 óbitos/ano, en tre os trabalhadores do setor formal seg u rados pelo SAT. O coeficien te médio de mortalidade, no mesmo período foi de 14,84 por 100.000 trabalhadores (MPS, 2003).

Assim, paradoxalmente, o SUS assume um pa pel social diferen ciado, ao se colocar, de fato, na atualidade, como a única política pública de cobertura universal, para o cuidado da saúde dos trabalhadores, obrigando a busca da redefinição das práticas de saúde e a con s trução de um "n ovo modelo" de atenção.
No final do ano de 2002, uma oportunidade política, surgida no âmbi to da Sec reta ria de Assistência à Sa ú dedo Ministéro da Saúde, permitiu a criação da Rede Nacional de Atenção In tegral à Saúde do Trabalhador (RENAST), pela porta ria 1.679 de 19 de setembro de 2002 . Apesar das críticas e dos de sen contros insti tucionais observados no processo de elaboração desse instrumento, em particular à ênfase nas ações assistenciais, a port a ria foi apoi ada pelos profissionais e técnicos dos CRST e setores do m ovim en to dos trabalhadores, que recon h eceram na iniciativa uma oportunidade de institucionalização e fortalecimento da Saúde do Trabalhador, no SUS. Pela primeira vez, seria possível contar com um financiamen to extrateto das ações, vinculado à operacionalização de um Plano de Trabalho de Saúde do Trabalhador, em nível estadual e municipal.

A partir de 2003, a coordenação da Área Técnica de Saúde do Trabalhador do Ministério da Saúde priorizou a implementação da RENAST como a principal estratégia da Política Nacional de Saúde do Trabalhador (PNST) para o SUS (MS, 2004). Para isto, foi organizado um su porte técnico regionalizad para ass ess orar o processo de implem entação da RENAST, pri oritariam en te nas regiões Norte, Norde s te e Centro-Oeste. Também, buscou-se ampliar e consolidar a articulação intra-setorial, no âmbito do Ministério da Saúde, em particular com a Atenção Básica; retomar o processo de discussão técnico - político de definição de uma Política de Sa ú de do Trabalhador para o SUS e de uma Política Nacional de Saúde do Trabalhador, de caráter intersetorial, envo lven do os Ministérios do Trabalho e Emprego e Previdência. Outra frente de atuação envolve a implem entação de um amplo processo de capacitação, nos Estados e municípios, de modo articulado com a Secretaria de Gestão do Trabalho em Saúde (SGETES) e a participação dos pólos de educação permanente, de universidades e insti tuições de ensino. Nesse senti do, está sendo organizada uma rede de centros colaboradores em Saúde do Trabalhador no SUS, para apoiar essa capacitação, forn ecer assessoria técnica e produzir conhecimen tos e material de apoio necessários ao pleno desenvolvimen to das atividades.

No capítulo dos instrumentos e normas, a portaria MS 777, de 28 de abril de 2004, dispõe sobre os procedimentos técnicos para a notificação compulsória de agravos à saúde do trabalhador, em rede sentinela específica do SUS. 
Ela rei tera a importância da articulação intrasetorial na saúde, em particular as interfaces com as vigilâncias epidemiológica, sanitária e ambiental, de modo a suprir a necessidade de informação consistente e ágil sobre a situação da produção e o perfil de adoecimen to rel acionado ao trabalho. A portaria define 11 grupos de agravos, considerados de notificação compulsória na rede de serviços sentinela, em sintonia com os flu xos do Si s tema de In formação de Agravos de Notificação (SINAN). Para facilitar a operacionalização dessa portaria, estão s en do preparados pro tocolos técnicos para cada um dos 11 grupos de agravos, com a colaboração de técnicos das universidades, dos CRST e instâncias do controle social. Essas informações são con s i deradas essenciais para subsidiar o con trole social e orientar as ações de saúde e as mudanças nos ambien tes e condições de trabalho.

Com base na experiência adquirida nesses dois anos e meio de implem entação da RENAST, o tex to da portaria 1.679 está sendo revisto. Entre as inovações propostas estão: a) a ampliação do número de CRST e de uma nova estrutu ra para a RENAST, que passa a ser organiz ada a partir da Coordenação Estadual de Sa úde do Trabalhador, dos CRST e da rede sentinela; b) mudanças no processo de habilitação dos CRST, cu ja gestão passa a ser tanto municipal quanto estadual; c) definição mais clara dos mecanismos de controle social, dos critérios de habilitação e acompanhamento dos CRST e do sistema de informação do SIA/SUS.

\section{A estratégia da RENAST para a atenção à Saúde do Trabalhador no SUS}

Conceitualmente, a RENAST é uma rede naci onal de informação e práticas de saúde, organizada com o propó sito de implementar ações assistenciais, de vigilância e de promoção da saúde, no SUS, na pers pectiva da Sa ú dedo Trabalhador. A compreensão do processo saúdedoença dos trabalhadores, que norteia a RENAST, está baseada no enfoque das relações Trabalho-Saúde-Doença e da centralidade do trabalho na vida das pessoas, desenvo lvi do pela epidemiologia social. A RENAST integra e articula as linhas de cuidado da atenção básica, da média e alta complexidade ambula torial, préhospitalar e hospitalar, sob o controle social, nos três níveis de gestão: nacional, estadual e municipal, ten do como ei xo os CRSTs. Estes deixam de ser porta de en trada do Si s tema e assumem o papel de su porte técnico e científico e de núcl eos irradiadores da cultura da cen tralidade do trabalho e da produção social das doen ças, no SUS. Além disso, são o lócus privilegi ado de articulação e pa ctuação das ações de saúde, in tra- e inters etorialmente, no seu território de abrangência, que pode ser a capital, á reas metropolitanas, municípios pólos de assistência das regiões e microrregiões de saúde.

Entre as tarefas a serem cumpridas pelos CRST estão: prover suporte técnico adequado às ações de assistência e vigilância da saúde do trabalhador; recolher, sistematizar e difundir in formações de modo a viabilizar as ações de vigilância, facilitar os processos de capacitação e educação perman en te para os profissionais e técnicos da rede do SUS e o con trole social.

Considerando a importância da produção, sistematização, análise e disseminação da informação em Saúde do Trabalhador, a RENAST prevê a or ganização da rede sentinela de notificação, que deverá cobrir os 5.230 municípios brasileiros. O termo sentinela é empregado, aqui, de modo distin to daquele utilizado pela epidemiologia para designar um even to ou problema de saúde em uma população ou comunidade. É utilizado para nomear a rede de serviços assistenciais de retaguarda, de média e alta complexidade, organizada de modo a gara $n$ tir a geração de informação e viabilizar a vigilância da saúde. Assim, o "s erviço sen tin ela" é responsável pela notificação e informações que irão gerar as ações de prevenção, vigilância e de in tervenção e mudanças, em Saúde do Traba$1 \mathrm{~h}$ ador.

Em situações especiais, caracterizadas a p a rtir da presença de fatores de ris co para a saúde nos processos produtivos, em um dado território e por critérios epidemiológicos, previdenciários, socioeconômicos, além da presença efetiva do con trole social, as ações de saúde do trabalhador poderão ser organizadas, no nível local, em municípios sentinelas. A utilização desse termo tem gerado confusão com o concei to de "município de alto ri s co", em pregado pela epidemiologia, estando em discussão, no processo de revisão da portaria.

Apesar da necessidade de se aclarar esses con ceitos, a organização da Rede Sentinela e dos Municípios Sentinelas tem o propósito de desenvolver metodologias e organizar o fluxo de informação e atendimento aos doentes e acidentados do trabalho, em todos os níveis de a tenção do SUS: na rede básica, média e alta 
com plexidade de modo arti c u lado com as Vigilâncias Sanitária, Epidemiológica e Ambiental.

Os princípios e diretrizes que norteiam a RENAST são coinciden tes com os da Política Nacional de Saúde do Trabalhador e podem ser resumidos em: I) a tenção integral à Saúde dos Trabalhadores; II) articulação intra- e intersetoriais; III) informações em Saúde do Trabalhador; IV) apoio ao de s envo lvi m en to de estudos e pe squisas; V) capacitação permanen te em Sa ú de do Trabalhador e VI) participação da comunidade na gestão das ações em Saúde do Trabalhador (MS-PNST, 2004).

A construção de ambien tes e processos de trabalho saudáveis; o fortalecimento da vigilância de ambientes, processos e agravos relacion ados ao trabalho e a assistência integral à saúde caracterizam a atenção integral à saúde. Pressupõem a adequação e ampliação da capacidade institucional de modo a permitir que este conjunto de ações seja desenvolvido com competência e resolutividade pela rede de serviços de saúde, em ação coordenada de diversos setores do governo e da sociedade civil. A articulação intra-setorial envolve todas as instâncias do SUS. NoMinistério da Saúde, a Áre a Técnica de Sa ú de do Trabalhador é o pon to focal dessa articulação e abrange, no campo da assistência, a Atenção Básica, a Média e Alta Complexidade e os programas e ações direcio$\mathrm{n}$ adas a gru pos pop ulaci onais específicos, en tre eles: as mulheres, os idosos; criança e adolescente; portadores de necessidades especiais. Também é estratégica a articulação das ações das Secretarias de Vigilância em Saúde, em especial com a Coordenação Geral de Vigilância Am bi ental (CGVAM); de Gestão do Trabalho e da Educação na Saúde; de Gestão Participativa; de Ciência, Tecnologia e In sumos Estratégicos; além da Anvisa e Funasa. Entre os fóruns estratégi cos para a pactuação in tra-setorial estão: o Conselho Nacional de Sa ú de (CNS); a Comissão In ter-s etorial de Saú de do Trabalhador (CIST); o Colegi ado de Secretários do Ministério da Saúde; a Comissão Permanen te de Saúde, Ambien te, Produção e De s envo lvimento Sustentável (Copesa); o Con selho Naci onal de Sec retários de Sa ú de (Conass) e o Conselho Nacional de Secretários Municipais de Saúde (Conasems). A articulação inter-setorial tem como interlocutores privilegiados os Ministérios do Trabalho e Emprego, Previdência Social e Meio Ambien te e está sob a responsabilidade direta do Gru po Exec utivo Interministerial em Saúde do Trabalhador (GEISAT). O utros fóruns de arti- culação inters etorial são: o Fórum Perma nen te de Erradicação do Trabalho Infantil; Con selho Nacional de Segurança Química (Conasq); Conselho Nacional de Direi tos Humanos; Comissão Nacional de Bi oss egu rança (CTNBIO) e os Movimentos Sociais e Organizações Sindicais de trabalhadore s. Con si dera n do a importância da produção de conhecimen to para o desenvolvi m en to da atenção à Sa ú de do Trabalhador, particularmen te nesse cen á rio de mudanças assinalado anteri ormen te, ela deve estar orientada por crité rios epidemiológicos, relevância social e capacidade das insti tuições de pes quisa e envo lver todos os equ i p a m en tos da rede de serviços do SUS, em particular os CRST. A capacitação dos profissionais, o utra prioridade para a implementação da RENAST, deve contemplar a divers i $\mathrm{d}$ ade e especificidades regionais, incorporar os princípios do trabalho cooperativo, in terdisciplinar e em equipe multi-profissional e a ex periência acumulada pelos Estados e municípios.

A participação dos trabalhadores é considerada essencial para a identificação dos fatores de risco presen tes no trabalho e das repercussões destes sobre o processo saúde-doença. Também é fundamental para a transformação das condições geradoras de aci den tes e doen ça . $\mathrm{Na}$ atualidade, o crescimen to das relações informais e precárias de trabalho exige a criação ou identificação de novas modalidades de representação dos trabalhadores, para além das organizações sindicais tradicionais (Silveira, Ribeiro \& Lino, 2005).

Sobre a Informação em Saú de do Trabalhador, além dos aspectos já mencion ados, a Política do Ministério da Sa ú de para a Informação e Informática estabelece o propósito de: promover o uso inovador, criativo e transformador da tecnologia da informação, para melhorar os processos de trabalho em saúde, resultando em um Sistema Nacional de Info rmação em Saúde articulado, que produza informações pa ra a ge stão, a prá tica profissional, a geração de conhecimentos e o controle social, garantindo ganhos de eficiência e qualidade mensuráveis através da ampliação de acesso, equidade, integralidade e humanização dos serviços. A criação do Observatório Nacional de Saúde do Trabalhador, com o apoio da repres entação da OPAS no Brasil, visa facilitar a monitorização e análise da situação de saúde dos trabalhadores, tomando por base as recom endações do Comitê Temático In terdisciplinar da Rede In teragencial de Informações para a Sa ú de (RIPSA), in corporado 
ao Sistema de Informações de Agravos Notificá veis (Sinan).

O financiamento das ações de Saúde do Trabalhador no SUS é outra questão complexa e ainda não resolvida. A implementação da RENAST conta, nessa fase inicial, com recursos ex tra-teto, transferi dos pelo Fun do Nacional de Saúde aos Estados e municípios. Esses recursos permiti ram a habilitação inicial de 11 CRSTs em 2002, a mpliadbs para 111, em ago sto de 2005. Nesse período, foram liberados recursos da ordem de 30 milhões de reais, cifra s em preceden tes para o financiamen to da Sa úde do Trabalhador no SUS.

\section{Ca racterísticas do "m odelo de atenção" da RENAST}

Para Paim (1999) modelos assistenciais são co m binações tecnoló gicas estruturadas pa ra o enfrentamen to de problemas de saúde individual e coletiva em determinados espaços-populações, incluindo ações sobre o ambiente, grupos populacionais, equipamentos comunitários e usuários de serviços de saúde (...). O autor assinala que não são normas ou exemplos a serem seguidos, mas do uso de racionalidades diversas paraapráxis.

A ri gor não se pode falar de um "modelo de a tenção da RENAST", uma vez que este é o próprio modelo de atenção do Sis tema Único de Saúde, organizado segundo os princípios da un ivers a lid adede acesso, integralidade da atenção com equidade e controle social, em um dado território, de modo regionalizado e hierarquizado, privilegiando a estratégia da Atenção Básica de Saúde e o enfoque da Promoção da Saúde.

Os trabalhadores sempre foram usuários do sistema de saúde. A proposta da RENAST é qualificar essa atenção, fa zen do com que o sistema, como um todo, entenda e funcione na perspectiva da Saúde do Trabalhador. Pa ra o trabalhador, na qualidade de indivíduo, ainda que os procedimentos diagnósticos e o tratamento da doença que apres enta sejam os mesmos, indepen den temente, de o agravo estar ou não relacion ado ao seu trabalho atual ou pregresso, é importan te que esta relação seja estabel ecida e feitos os encaminhamentos adequados. Somente a partir do estabelecimen to da relação entre o agravo ou doença com o trabalho e do registro no sistema de informação é possível coletivizar o fen ô meno e desencadear proced $\mathrm{i}$ men tos de vigilância que levem à mudança nas condições e ambientes de trabalho geradoras de doença. Por outro lado, a iden tificação de situações ou de fatores de ris co para a saúde nas situações de trabalho, originada nas ações de vigilância, permite o encaminhamentodos expo s tos e doen tes à assistência adequada. Assim, as ações de Saúde do Trabalhador são desencade adas a partir da iden tificação de um agravo à saúde ou de uma situação de risco, relacionados ao trabalho. A indissociabilidade das ações assistenciais e de vigilância da saúde constitui uma pedra angular da Sa ú de do Trabalhador.

A Atenção Básica é a principal referência para o re-ordenamento da atenção à saúde na atualidade. Constitui a porta de entrada dos serviços de saúde e apresenta-se como a melhor es tra té giapara otimizar a saúde da população e minimizar as desigualdades entre os grupos populacionais, de modo a se alcançar equidade. Além de permitir a entrada no sistema, ela propicia atenção com resolutividade e não direcionada exclusivamente para a doença e coordena ou integra a referência a níveis mais complexos. É or ganizada a partir de uma base territorial e critérios epidemiológicos, pri orizando os problemas mais comuns em uma dada comunidade, oferecendo serviços de prevenção, cura e reabilitação, visandomaximizar a saúde e o bem-estar das pessoas. Pode-se dizer que a inserção efetiva das ações de Saú de do Trabalhador no SUS está diretamente relacion ada à possibilidade de sua assimilação pela atenção básica. Além dos fatores or ganizacionais mencionados anteri ormente, o crescimen to do trabalho informal, familiar e em domicílio, reforç a o papel da atenção básica de fazer ch egar ações de saúde o mais próximo possíveldeonde as pessoas vivem e trabalham. Oacolhimen to dos trabalhadores na porta de entrada do sistema, a investigação do trabalho como fator determinante dos processos saúde - doença e avaliação e manejo das situações de risco no trabalho, incorpora n do o saber do trabalhador, s ob o controle social, são possibilidades concretas na a tenção básica. Entretan to, para que isto se efetive, não basta acre s centar mais uma atribuição às já sobrecarregadas equipes de trabalho. È importan te que as tarefas sejam redefinidas e redimensionadas, as equipes capacitadas e garantidos os procedimentos de referência e contra-referência.

O "m odelo de atenção" da Saúde do Trabalh ador, orientado pela Promoção da Saúde privilegia as ações sobre as condições de vida das pessoas e do coletivo, no território on de vivem e trabalham. A Política Naci onal de Promoção 
da Sa ú de focaliza a con tri buição dos modos de viver, ou seja, os modos como sujeitos e coletivos el egem determinadas opções de viver como desejáveis, o rganizam suas escolhas e criam possibilidades pa ra satisfazer suas necessidades, desejos e interesses pertencem à ordem coletiva, uma vez que seu processo de construção dá-se no co n texto da própria vida, envolvendo forças politicas, econômicas, afetivas, cultu rais e sociais existentes num território local, regional, nacional e/ou global na produção do processo saúde-doença. (MS, 2005). O utra con tri buição importante do en foque da Promoção da Sa ú de se refere ao empoderam ento dos trabalhadores para tom ada de decisão e gerenciamento de suas condições de saúde. Isto pre s supõe a ga rantia de informação e meios efetivos para esse gerenciamento que coi n ci de com a "participação e não del egação", uma das "idéias for ça" da Sa ú de do Trabalhador.

Finalizandoesta breve iden tificação dos pilares que orientam o desenho e a atuação da RENAST, é importante destacar a idéia-proposta ou con ceito de Desenvo lvimento Humano Sustentável. Apesar das controvérsias que envolvem o termo e as formas como essa expressão tem sido apropri ada por distintos setores sociais, a realidade tem apon tado, de modo con sistente, a necessidade de inclusão das qu e stões relacionadas às relações Trabalho-Ambiente e Saúde, de modo especial a Saúde do Trabalhador, nas agendas de discussão do modelo de des envo lvim en to a ser ado tado no país (Rigotto, 2003). O que produzir? Como? Quem? Para que? Como repartir os ônus e as benesses do produto desse trabalho? São questões cruciais cujas respostas ex i gem o envo lvim en to de toda a sociedade.

$\mathrm{Na}$ atualidade, as políticas públicas setoriais que en focam a produção e distri buição de ben $s$ oriu n dos da tra $\mathrm{n}$ s formação da natu reza e pre stação de serviços, na agricultura, comércio, indústria, de s envo lvim en to e ciência e tecnologia não têm, via de regra, levado em consideração as conseqüências sobre as condições de vida e os perfis de adoecimento relacionados ao trabalho. A mesma fragm entação se reproduz nas políticas setoriais na esfera da Previdência Social, Meio Am biente e Trabalho. Pa ra mudança desse quadro, é necessário que as políticas públicas privilegi em a construção de processos produtivos limpos e saudáveis, de modo a diminuir os riscos de adoecimento e a degradação do ambien te e garantir uma distribuição eqüitativa e justa dos benefícios e problemas gerados nos processos produtivos.
A figura 1 tenta re sumir o "modelo de atenção" da RENAST.

Esquematicamente, as ações de Saúde do Trabalhador compreendem a assistência aos agravos, a vigilância dos ambi en tes e condições de trabalho (Vigilância Sanitária), da situação de saúde dos trabalhadores (Vigilância Epidemiológica) e da situação ambi ental (Vigilância Ambiental). Estão incluíd as, ainda, a produ çã o, coleta, sistematização, análise e divulgação das informações de saúde, a produção de conheci$m$ en to e as ativid ades educativas, todas elas desenvo lvidas sob o controle da sociedade organizada. A partir das ações assistenciais são iden tificados os "casos" ou situações de adoec im en to relacion ado ao trabalho, que são notific ados ao Si s tema de Informação. A partir dessa informação são de sen cadeados os proced i m entos de vigilância da saúde. Na outra ponta, as ações de vigilância dos ambientes e condições de trabalho, de vigilância epidemiológica de agravos e da vigilância ambiental também geram informação e identificam "casos de doentes ou de suspeito s" que são encaminhados à rede de serviços de assistência. Fecha-se, dessa forma, o ciclo de atenção integral à saúde dos trabalhadores, que inclu em ainda, procedimentos de Promoção da Saúde definidos e implementados no âmbi to do sistema de saúde e fora dele, pelo setor Trabalho, Previdência Social, Meio Ambien te e setores de governo responsáveis pelas políticas de desenvo lvimen to econ ômico e social.

\section{Desafios e perspectivas para a RENAST}

Apesar dos percalços, pode-se con si derar que a estratégia da RENAST, para prover uma atenção diferenciada aos trabalhadores no SUS tem sido bem sucedida. Hoefel, Dias \& Silva (2005) destacam, en tre as dificuldades a serem superadas: a) a lógica do modelo da RENAST, cen trada na atuação regionalizada dos CRSTs, não corre s pon de à or ganização do SUS cen trada na municipalização; b) frágil articulação intra-setorial uma vez que a Sa ú de do Trabalhador ainda não foi efetivamen te incorporada na Agenda de Saúde do SUS, nos níveis federal, estadual e municipal; c) confusão dos papéis das coordenações estaduais e municipais de Saúde do Trabalhador e dos Cen tros de Referência; d) falta de ori entação dara quanto aos processos de pa ctu ação dos proced im en tos de assistência, vigilância e informação em saúde, uma vez 


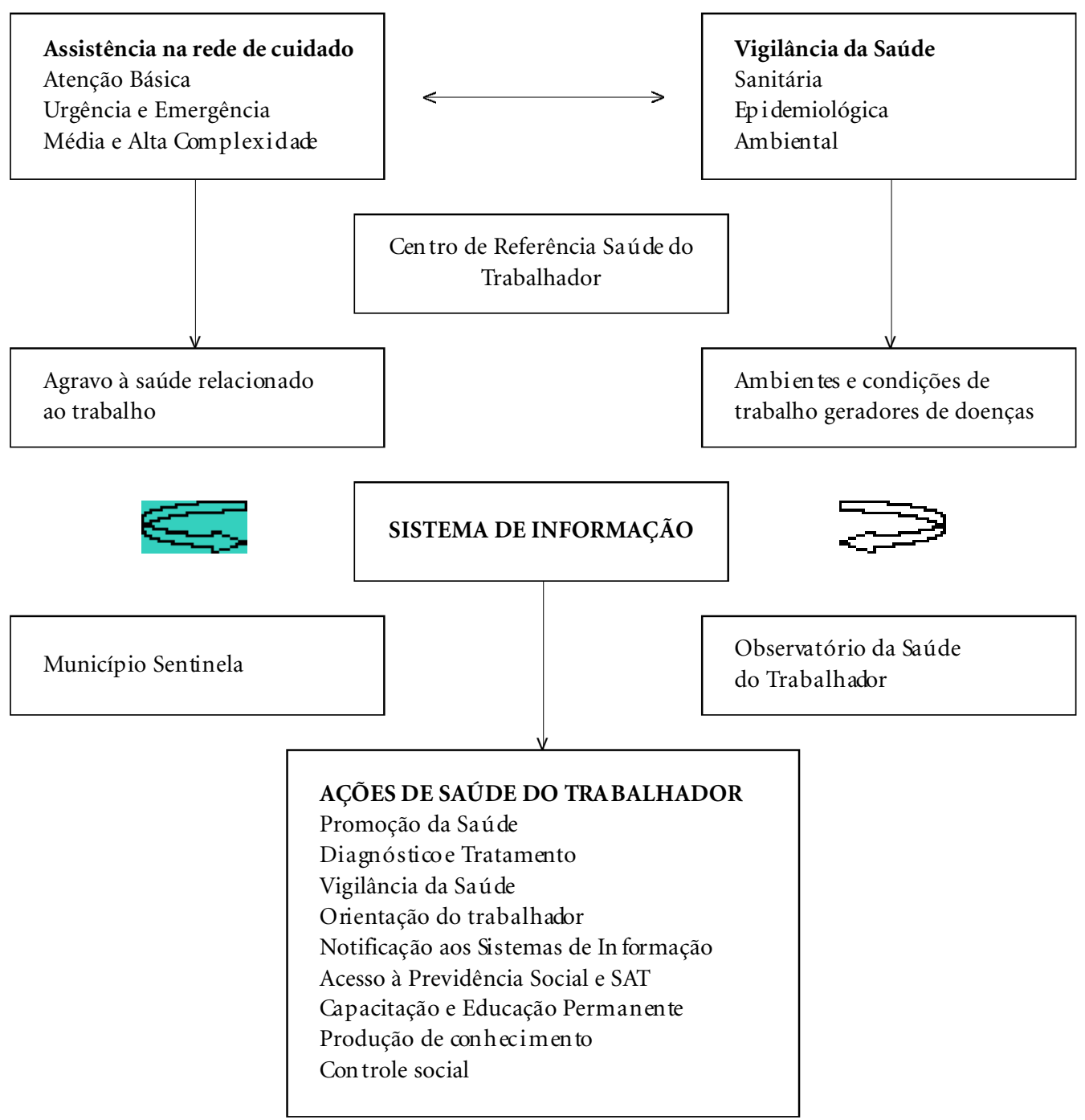

que, com freqüência, as áreas de atuação dos CRSTs não coincidem com as Regionais de Sa úde; e) o desprep a ro dos profissionais de saúde que atuam na rede de serviços para lidar com os ris cos e agravos à saúde, relacion ados com o trabalho e definir os encaminhamen tos médicos e administrativos adequ ados; f) o controle s ocial está fragilizado, em decorrência das mudanças na or ganização sindical e do gra ndenúmero de trabalhadores no merc ado de trabalho formal, que ainda não se organizaram para participar dos fóruns sociais; g) desvio dos recursos destinados à implantação da RENAST para cobrir outras necessidades no âmbito do sistema de saúde; h) apesar dos avanços regionais, ainda se observa o precedência da assistência em detrimen to das ações de vigilância, prejudicando a integralidade das ações; i) as ações intersetoriais ainda são tímidas e localizadas.

O mom en to históri co e o processo de criação da RENAST permitem iden tificá-la como uma política compensatória, lamentavelmente, focalizadae desvinculada de outras políticas de seguridade social. Foi implementada por um Estado cuja atuação esteve marc ada pela res tri- 
ção de direi tos dos trabalhadbres e outras mudanças estruturais destinadas a diminuir os gastos públi cos para gerar superávit destinado ao paga m en toda dívida ex tern a

En tret an to a RENAST também é uma política tra $\mathrm{n}$ s formadora e de inclusão, pois tem possibilitado dar visibilidade às questões de Saúde do Trabalhador nos serviços de saúde; facilitado o diálogo com os gestores e o planejam en to de ações, financiadas por rec u rsos ex tra-teto. $\mathrm{O}$ processo de mobilização social expresso na re alização de cerca de 500 con ferências municipais e estaduais de Saúde do Trabalhador, na etapa preparatória da 3a CNST, prevista para novembro deste ano, também pode ser creditado ao trabalho da RENAST. Assim, é importante re afirmar os ganhos e as possibilidades da RENAST e, s em ufanismo, en frentar as dificuldades e solucionar, na medida do possível, os impasses e as lacunas ob servadas.

O movim en to da Sa ú de do Trabalhador em nosso país está próximo de completar 30 anos. Nesse período, enfren tou inúmeras adversidades, mas persiste apoiado nos sonhos e no trabalho de mu i tos que acreditam que o Trabalho é meio de Vida.

\section{Colaboradores}

EC Dias e MG Hoefel, em conju n to, conceberam e planejaram o arti go, realizaram a discussão e aprimoram en to do tex to. EC Dias redigiu o trabalho e ambas as autoras realizarm sua revisão final.

\section{Referências bibliográficas}

Augu s toLGS 1985. Ben zolismo em uma sider ú r gica. Rev. SOS 10:153-87.

Berlinguer G 1983. A saúde nas fábricas. CEBES-HucitecOboré, São Paulo.

Costa DF et al. 1989. Programa de Saúde dos Trabalhadores - a experiên ciada Zona Norte: uma alternativa em Saúde Pública. Oboré, São Paulo.

Dias EC 1994. Atenção à saúde dos trabalhadoresno setor saúde (SUS), no Brasil:realidade, fantasia ou utopia? Tese de do utorado. Departamen to de Medicina Preven tiva e Social, FCM/Unicamp Campinas.

Hoefel MG, Dias EC \& Si lva JM 2005. A atenção à Saúde do Tra balhador no SUS: a proposta de consti tuição da RENAST. Brasília, Mnistério da Saúde. Disponível em<3cnst@saude.gov.br $>$

Lacaz FAC 1996. Saúde do Trabalhador: um estudo sobre as fo rmações disc u rsivas da academia, dos serviços e do movimento sindical. Tese de doutorado. Departamen to de Medicina Preventiva e Social, FCM-Unicamp. Campinas.

Men des R 1988. Subsídios para um debate em torno da revisão do atual modelo de organização da Saúde Ocupacional no Brasil. Rev. Bra silei radeSaúde Ocupacional 64(16):7-21. 
Men des R \& Dias EC 1991. Da medicina do trabalho a Sa ú de do Trabalhador. Rev. Saúde Públ. 25(5):341 349.

Merhy E, Cecílio LCO \& Nogu eira RC 1992. Por um modelo tecno-assistencial da politica de saúde em defesa da vida: contribuições pa ra as Conferências de Saúde. Mnistério da Saúde, Brasília. ( Cadernos da 9a Conferência Nacional de Saúde. Descentralizando e Democratizandoo Con h ecimen to, v. 1).

Mnistério da Previdência Social. Mi nuta de Política Nacional de Segurança e Saúde do Trabalhador. Portaria Interministerial no 800, de 3 de maio de 2005, dos ministros de Estado da Previdência Social, da Saúde e do Trabalho e Emprego. DOU. Número 85 de 05/05/ 2005. Dis ponível em <www.previdênciasocial.gov.br >

Mnistério da Saúde, Fundação Oswaldo Cruz 1987. Relatório Final da 1a Con ferên cia Nacional de Saúde dos Trabalhadores. Brasília, Ministéró da Saúde/FiocruzENSP-CESTEH.

Mnistério da Saúde-Secretaria Nacional de Vigilância Sanitária 1991. Saúde do Trabalhador: Diretrizes de Ação para o SUS. Brasília (mimeo).

Ministério da Sa ú de 2002. Con ferência Naci onal de Sa úde do Trabalhabr 2. Brasília, DF 1994. Anais. Brasília. Mnistério da Saúde. (Série D. Reuniões e Conferências).

Mnistério da Saúde 2004. Política Na cional de Saúde do Tra balhador pa ra o SUS. Brasília (mimeo).
Ministério da Sa ú de2005. Política Na cional de Promoção da Saúde. MS, Brasília. Disponível em <www.saude. gov.br $>$. Ace s sado em 20 de julho de 2005.

Od done I et al. 1986. Ambiente de tra balho: a luta dos trabalhadores pela saúde. Hucitec, São Paulo.

Paim JS 1999. Políticas de descentralização e atenção primária a saúde, pp. 489-503. In Z Ro u qu ayrol \& N Almeida. Epidemiologia e saúde. 5a ed. Medsi, Rio de Jan eiro.

Pimenta AL \& Capistrano Filho D (or gs.) 1988. Saúde do Trabalhador. Hucitec, São Paulo.

Ribei ro HP \& Lacaz FAC (orgs.) 1984. De que adoe cem e morremostrabalhadores. DIESAT, São Paulo.

Rigo t to RM 2003. Saúde Ambiental \& Saúde dos Traba1h adores: uma aproximação promissora en tre o Verde e o Vermelho. Revista Brasilei ra de Epidemiologia 4(6): 388-404.

Silveira AM, Ribeiro FSN \& Lino AFPF 2005. O con trole social no SUS e a RENAST. 3a Conferência Nacional de Saúde do Trabalhador. 3a CNST: Trabalhar sim! Adoecer não! Coletânea de Texto. Mnistério da Sa úde, Mnistério do Trabalho e Emprego, Mnistério da Previdência e Assistência Social. Brasília. Mnistério daSa úde.

Artigo apresen t ado em 10/08/2005

Aprovado em 31/08/2005

Versão final apresen tada em 8/09/2005 